Median CIT was significantly lower for the timesheet period than for the preceding 2-year period (13.27 h vs $21.45 \mathrm{~h} ; P<0.001)$. In addition, the incidence of delayed graft function was lower in the timesheet period $(20.7 \%$ vs $34.7 \% ; P=0.011$ ). The authors attribute the difference in CIT to the effect that timesheet keeping had on the motivation levels of personnel involved in the transplantation procedures. They do not report any specific changes made to systems at their center on the basis of the timesheet analysis; however, they identify the availability of an operating room as the major limiting factor for short CIT and suggest that, if availability could be improved, CITs at their institution could be reduced to as little as $9 \mathrm{~h}$.

The authors also note that, at their institution, median CIT for transplantations affected by the French national organ sharing program ( $15.7 \%$ of procedures during the timesheet period) was twice as long as the CIT for those without national priority. They propose that the impact of national organ sharing on outcomes of transplantation should be regularly evaluated.

Original article Vacher-Coponat $\mathrm{H}$ et al. (2007) Cold ischemia time in renal transplantation is reduced by a timesheet in a French transplant center. Transplantation 83: 561-565

\section{Reprocessing of formalin-fixed tissue for EM precludes accurate TBMN diagnosis}

Glutaraldehyde-fixed, plastic-embedded tissue is preferred for electron microscopy (EM)-based diagnostic renal pathology. If no glomeruli are present on glutaraldehyde-fixed sections, tissue originally fixed in formalin and embedded in paraffin for light microscopy can be deparaffinized and embedded in plastic resin for EM. A recent report shows, however, that reprocessing tissue in this way can cause artifactual thinning of the glomerular basement membrane (GBM) that prevents accurate diagnosis of thin basement membrane nephropathy (TBMN).

Formalin-fixed, paraffin-embedded tissue blocks from 21 renal biopsies (8 TBMN, 6 minimal-change disease, 2 histologically normal, and 5 diabetic nephropathy) were reprocessed, and examined using EM. Glutaraldehyde-fixed, plastic-embedded tissue from all 21 biopsies had previously been examined by EM. For each biopsy, 80 measurements of the GBM were made on glutaraldehyde-fixed tissue, and 80 on deparaffinized formalin-fixed tissue.

Reprocessing of formalin-fixed tissue resulted in considerable GBM thinning in all 21 cases. The mean decreases in GBM thickness with formalin-fixed tissue compared with glutaraldehyde-fixed tissue were $23 \%$ $(P=0.004)$ for TBMN biopsies, $40 \%(P=0.004)$ for normal and minimal-change disease biopsies, and 34\% $(P=0.031)$ for diabetic nephropathy biopsies. On deparaffinized formalin-fixed tissue, four of the six minimalchange disease cases had a mean GBM thickness below the laboratory's threshold for TBMN diagnosis ( $<250 \mathrm{~nm}$ for males and $<220 \mathrm{~nm}$ for females), and would have been incorrectly diagnosed as TBMN. No clear criteria could be identified for accurate TBMN diagnosis in formalin-fixed tissue.

Original article Nasr SH et al. (2007) Thin basement membrane nephropathy cannot be diagnosed reliably in deparaffinized, formalin-fixed tissue. Nephrol Dial Transplant 22: $1228-1232$

\section{Mycophenolate mofetil treatment for resistant primary glomerulonephritis}

Mycophenolate mofetil (MMF) treatment might be useful for glomerulonephritis, but studies have produced conflicting results. Segarra et al. investigated the safety and efficacy of MMF in patients with resistant primary glomerulonephritis.

The study included 98 patients with different types of primary glomerulonephritis. All patients were resistant to recommended treatments (e.g. ciclosporin, cyclophosphamide, steroids) and none had received immunosuppressant drugs or NSAIDs for at least 6 months before enrollment. MMF was given for 12 months, with dose administered dependent on renal function $(2.0 \mathrm{~g} /$ day for patients with creatinine clearance $\geq 60 \mathrm{ml} / \mathrm{min} ; 1.5 \mathrm{~g} /$ day for those with creatinine clearance $<60 \mathrm{ml} / \mathrm{min}$ ). In patients with evidence of complete or partial remission of proteinuria, MMF dose was tapered until treatment discontinuation or relapse. During (and from 6 months before) the study, patients followed a low-salt diet and received angiotensin-converting-enzyme inhibitors or angiotensin receptor blockers. 